\title{
DYNAMIC MODEL AND PHASE TRANSITIONS FOR LIQUID HELIUM
}

\author{
TIAN MA AND SHOUHONG WANG
}

\begin{abstract}
This article presents a phenomenological dynamic phase transition theory - modeling and analysis - for superfluids. As we know, although the time-dependent Ginzburg-Landau model has been successfully used in superconductivity, and the classical Ginzburg-Landau free energy is still poorly applicable to liquid helium in a quantitative sense. The study in this article is based on 1) a new dynamic classification scheme of phase transitions, 2) new time-dependent Ginzburg-Landau models for general equilibrium transitions, and 3) the general dynamic transition theory. The results in this article predict the existence of a unstable region $H$, where both solid and liquid He II states appear randomly depending on fluctuations and the existence of a switch point $M$ on the $\lambda$ curve, where the transitions changes types.
\end{abstract}

\section{INTRODUCTION}

Superfluidity is a phase of matter in which "unusual" effects are observed when liquids, typically of helium- 4 or helium-3, overcome friction by surface interaction when at a stage, known as the "lambda point" for helium-4, at which the liquid's viscosity becomes zero. Also known as a major facet in the study of quantum hydrodynamics, it was discovered by Pyotr Leonidovich Kapitsa, John F. Allen, and Don Misener in 1937 and has been described through phenomenological and microscopic theories.

Atoms helium have two stable isotopes ${ }^{4} \mathrm{He}$ and ${ }^{3} \mathrm{He} .{ }^{4} \mathrm{He}$ consists of two electrons, two protons and two neutrons, which are six fermions. Therefore, ${ }^{4} \mathrm{He}$ has an integral spin and obey the Bose-Einstein statistics. Liquid ${ }^{4} \mathrm{He}$, called the Bose liquid, displays a direct transition from the normal liquid state (liquid He I) to the superfluid state (liquid He II) at temperature $T=2.19 \mathrm{~K}$, which can be considered as the condensation of particles at simple quantum state.

The main objectives of this article are 1) to establish a time-dependent GinzburgLandau model for liquid ${ }^{4} \mathrm{He}$, and 2) to study its dynamic phase transitions. Hereafter, we shall present briefly the main ingredients of the study presented in this article. The ideas and method in this article can be used to study ${ }^{3} \mathrm{He}$ and its mixture with ${ }^{4} \mathrm{He}$; this study will be reported elsewhere.

First, in the late 1930s, Ginzburg-Landau proposed a mean field theory of continuous phase transitions. With the successful application of the Ginzburg-Landau

1991 Mathematics Subject Classification. 76A25, 82B, 82D, 37L.

Key words and phrases. helium-4, dynamic phase transition, lambda point, time-dependent Ginzburg-Landau models, dynamic transition theory.

The work was supported in part by the Office of Naval Research and by the National Science Foundation. 
theory to superconductivity, it is nature to transfer something similar to the superfluidity case, as the superfluid transitions in liquid ${ }^{3} \mathrm{He}$ and ${ }^{4} \mathrm{He}$ are of similar quantum origin as superconductivity. Unfortunately, we know that the classical Ginzburg-Landau free energy is poorly applicable to ${ }^{4} \mathrm{He}$ in a quantitative sense, as described in by Ginzburg in [1].

The starting point of the modeling used in this article is to introduce a general principle, based on the le Châtelier principle and some general characteristics of pseudo-gradient flow systems. This general principle leads to a unified approach to derive Ginzburg-Landau type of time-dependent models for equilibrium phase transitions. With this general principle in our disposal, we derive some dynamic models for superfluid transitions.

In addition, an important difference for the new dynamic models introduced here in this article from the classical ones is based on the separation of superfluid and normal fluid densities as described in (3.1) and their interactions in the GinzburgLandau free energies.

Second, classically, phase transitions are classified by the Ehrenfest classification scheme, based on the lowest derivative of the free energy that is discontinuous at the transition. The superfluid phase transitions has been regarded as continuous phase transitions, also called second-order phase transitions. However, many important issues are still still not clear; see among many others Ginzburg [1], Reichl [7] and Onuki [6].

One important new ingredient for the analysis is a new dynamic transition theory developed recently by the authors [3, 2]. With this theory, we derive a new dynamic phase transition classification scheme, which classifies phase transitions into three categories: Type-I, Type-II and Type-III, corresponding respectively to the continuous, the jump and mixed transitions in the dynamic transition theory.

The results in this article lead to two physical predictions: 1) the existence of the unstable region $H$, where both solid and liquid He II states appear randomly depending on fluctuations, and 2) the existence of a switch point $M$, where the transitions, between superfluid state (liquid He II) and the normal fluid state (liquid He I), changes from first order (Type II with the dynamic classification scheme) to second order (Type-I). Of course, these predictions need to be verified by experiments, and it is hoped that the new model, the ideas and methods introduced in this article will lead to some improved understanding of superfluidity.

This article is organized as follows. First, the new dynamic transition theory is recapitulated in Section 2, and a dynamic phase transition model for ${ }^{4} \mathrm{He}$ is introduced in discussed in Section 3. The dynamic transitions of the the model is analyzed in Section 4. Section 5 gives some physical conclusions and predictions.

\section{General Principles of Phase Transition Dynamics}

In this section, we introduce a new phase dynamic transition classification scheme to classify phase transitions into three categories: Type-I, Type-II and Type-III, corresponding mathematically continuous, jump mixed transitions, respectively.

2.1. Dynamic transition theory. In sciences, nonlinear dissipative systems are generally governed by differential equations, which can be expressed in the following abstract form Let $X$ and $X_{1}$ be two Banach spaces, and $X_{1} \subset X$ a compact and dense inclusion. In this chapter, we always consider the following nonlinear 
evolution equations

$$
\frac{d u}{d t}=L_{\lambda} u+G(u, \lambda), \quad u(0)=\varphi
$$

where $u:[0, \infty) \rightarrow X$ is unknown function, and $\lambda \in \mathbb{R}^{1}$ is the system parameter.

Assume that $L_{\lambda}: X_{1} \rightarrow X$ is a parameterized linear completely continuous field depending contiguously on $\lambda \in \mathbb{R}^{1}$, which satisfies

$$
\begin{array}{ll}
L_{\lambda}=-A+B_{\lambda} & \text { a sectorial operator, } \\
A: X_{1} \rightarrow X & \text { a linear homeomorphism, } \\
B_{\lambda}: X_{1} \rightarrow X & \text { a linear compact operator. }
\end{array}
$$

In this case, we can define the fractional order spaces $X_{\sigma}$ for $\sigma \in \mathbb{R}^{1}$. Then we also assume that $G(\cdot, \lambda): X_{\alpha} \rightarrow X$ is $C^{r}(r \geq 1)$ bounded mapping for some $0 \leq \alpha<1$, depending continuously on $\lambda \in \mathbb{R}^{1}$, and

$$
G(u, \lambda)=o\left(\|u\|_{X_{\alpha}}\right) \quad \forall \lambda \in \mathbb{R}^{1} .
$$

Hereafter we always assume the conditions 2.2 and 2.3 , which represent that the system 2.1 has a dissipative structure.

A state of the system 2.1 at $\lambda$ is usually referred to as a compact invariant set $\Sigma_{\lambda}$. In many applications, $\Sigma_{\lambda}$ is a singular point or a periodic orbit. A state $\Sigma_{\lambda}$ of (2.1) is stable if $\Sigma_{\lambda}$ is an attractor; otherwise $\Sigma_{\lambda}$ is called unstable.

Definition 2.1. We say that the system (2.1) has a phase transition from a state $\Sigma_{\lambda}$ at $\lambda=\lambda_{0}$ if $\Sigma_{\lambda}$ is stable on $\lambda<\lambda_{0}$ (or on $\lambda>\lambda_{0}$ ) and is unstable on $\lambda>\lambda_{0}$ (or on $\lambda<\lambda_{0}$ ). The critical parameter $\lambda_{0}$ is called a critical point. In other words, the phase transition corresponds to an exchange of stable states.

Obviously, the attractor bifurcation of 2.1 is a type of transition. However, bifurcation and transition are two different, but related concepts.

Let $\left\{\beta_{j}(\lambda) \in \mathbb{C} \mid j \in \mathbb{N}\right\}$ be the eigenvalues (counting multiplicity) of $L_{\lambda}$, and assume that

$$
\begin{array}{lll}
\operatorname{Re} \beta_{i}(\lambda) \begin{cases}<0 & \text { if } \lambda<\lambda_{0}, \\
=0 & \text { if } \lambda=\lambda_{0}, \\
>0 & \text { if } \lambda>\lambda_{0},\end{cases} & \forall 1 \leq i \leq m, \\
\operatorname{Re} \beta_{j}\left(\lambda_{0}\right)<0 & & \forall j \geq m+1 .
\end{array}
$$

The following theorem is a basic principle of transitions from equilibrium states, which provides sufficient conditions and a basic classification for transitions of nonlinear dissipative systems. This theorem is a direct consequence of the center manifold theorems and the stable manifold theorems; we omit the proof.

Theorem 2.1. Let the conditions (2.4) and (2.5) hold true. Then, the system (2.1) must have a transition from $(u, \lambda)=\left(0, \lambda_{0}\right)$, and there is a neighborhood $U \subset \bar{X}$ of $u=0$ such that the transition is one of the following three types:

(1) Continuous Transition: there exists an open and dense set $\widetilde{U}_{\lambda} \subset U$ such that for any $\varphi \in \widetilde{U}_{\lambda}$, the solution $u_{\lambda}(t, \varphi)$ of 2.1 satisfies

$$
\lim _{\lambda \rightarrow \lambda_{0}} \limsup _{t \rightarrow \infty}\left\|u_{\lambda}(t, \varphi)\right\|_{X}=0 .
$$


(2) Jump Transition: for any $\lambda_{0}<\lambda<\lambda_{0}+\varepsilon$ with some $\varepsilon>0$, there is an open and dense set $U_{\lambda} \subset U$ such that for any $\varphi \in U_{\lambda}$, $\limsup _{t \rightarrow \infty}\left\|u_{\lambda}(t, \varphi)\right\|_{X} \geq \delta>0 \quad$ for some $\delta>0$ is independent of $\lambda$.

(3) Mixed Transition: for any $\lambda_{0}<\lambda<\lambda_{0}+\varepsilon$ with some $\varepsilon>0$, $U$ can be decomposed into two open sets $U_{1}^{\lambda}$ and $U_{2}^{\lambda}$ ( $U_{i}^{\lambda}$ not necessarily connected): $\bar{U}=\bar{U}_{1}^{\lambda}+\bar{U}_{2}^{\lambda}, U_{1}^{\lambda} \cap U_{2}^{\lambda}=\emptyset$, such that

$$
\begin{array}{ll}
\lim _{\lambda \rightarrow \lambda_{0}} \limsup _{t \rightarrow \infty}\|u(t, \varphi)\|_{X}=0 & \forall \varphi \in U_{1}^{\lambda}, \\
\limsup _{t \rightarrow \infty}\|u(t, \varphi)\|_{X} \geq \delta>0 & \forall \varphi \in U_{2}^{\lambda} .
\end{array}
$$

With this theorem in our disposal, we are in position to give a new dynamic classification scheme for dynamic phase transitions.

Definition 2.1 (Dynamic Classification of Phase Transition). The phase transitions for (2.1) at $\lambda=\lambda_{0}$ is classified using their dynamic properties: continuous, jump, and mixed as given in Theorem 2.1. which are called Type-I, Type-II and Type-III respectively.

An important aspect of the transition theory is to determine which of the three types of transitions given by Theorem 2.1 occurs in a specific problem. A corresponding dynamic transition has been developed recently by the authors for this purpose; see [3. We refer interested readers to these references for details of the theory. Hereafter we recall a few related theorems in this theory used in this article.

FIRST, we remark here that one crucial ingredient for applications of this general dynamic transition theory is the reduction of (2.1) to the center manifold function. In fact, by this reduction, we know that the type of transitions for 2.1) at $\left(0, \lambda_{0}\right)$ is completely dictated by its reduction equation near $\lambda=\lambda_{0}$ :

$$
\frac{d x}{d t}=J_{\lambda} x+g(x, \lambda) \quad \text { for } x \in \mathbb{R}^{m},
$$

where $g(x, \lambda)=\left(g_{1}(x, \lambda), \cdots, g_{m}(x, \lambda)\right)$, and

$$
g_{j}(x, \lambda)=<G\left(\sum_{i=1}^{m} x_{i} e_{i}+\Phi(x, \lambda), \lambda\right), e_{j}^{*}>\quad \forall 1 \leq j \leq m .
$$

Here $e_{j}$ and $e_{j}^{*}(1 \leq j \leq m)$ are the eigenvectors of $L_{\lambda}$ and $L_{\lambda}^{*}$ respectively corresponding to the eigenvalues $\beta_{j}(\lambda)$ as in (2.4), $J_{\lambda}$ is the $m \times m$ order Jordan matrix corresponding to the eigenvalues given by (2.4), and $\Phi(x, \lambda)$ is the center manifold function of 2.1 near $\lambda_{0}$.

SECOND, let 2.1) be a gradient-type equation. Under the conditions 2.4 and 2.5), in a neighborhood $U \subset X$ of $u=0$, the center manifold $M^{c}$ in $U$ at $\lambda=\lambda_{0}$ consists of three subsets

$$
M^{c}=W^{u}+W^{s}+D,
$$

where $W^{s}$ is the stable set, $W^{u}$ is the unstable set, and $D$ is the hyperbolic set of 2.6. Then we have the following theorem.

Theorem 2.2. Let (2.1) be a gradient-type equation, and the conditions (2.4) and (2.5) hold true. If $u=0$ is an isolated singular point of (2.1) at $\lambda=\lambda_{0}$, then we have the following assertions: 
(1) The transition of (2.1) at $(u, \lambda)=\left(0, \lambda_{0}\right)$ is continuous if and only if $u=0$ is locally asymptotically stable at $\lambda=\lambda_{0}$, i.e., the center manifold is stable: $M^{c}=W^{s}$. Moreover, 2.1) bifurcates from $\left(0, \lambda_{0}\right)$ to minimal attractors consisting of singular points of 2.1.

(2) If the stable set $W^{s}$ of (2.1) has no interior points in $M^{c}$, i.e., $M^{c}=$ $\bar{W}^{u}+\bar{D}$, then the transition is jump.

THIRD, we also a dynamic transition theorem of $(2.1)$ from a simple critical eigenvalue, which has been used in analyzing PVT systems and the ferromagnetic systems [4, 5]. We refer the interested readers to these references for details for this theorem.

2.2. New Ginzburg-Landau models for equilibrium phase transitions. In this section, we recall a new time-dependent Ginzburg-Landau theory for modeling equilibrium phase transitions in statistical physics.

Consider a thermal system with a control parameter $\lambda$. The classical le Châtelier principle amounts to saying that for a stable equilibrium state of a system $\Sigma$, when the system deviates from $\Sigma$ by a small perturbation or fluctuation, there will be a resuming force to restore this system to return to the stable state $\Sigma$.

By the mathematical characterization of gradient systems and the le Châtelier principle, for a system with thermodynamic potential $\mathcal{H}(u, \lambda)$, the governing equations are essentially determined by the functional $\mathcal{H}(u, \lambda)$. When the order parameters $\left(u_{1}, \cdots, u_{m}\right)$ are nonconserved variables, i.e., the integers

$$
\int_{\Omega} u_{i}(x, t) d x=a_{i}(t) \neq \text { constant. }
$$

then the time-dependent equations are given by

$$
\begin{aligned}
& \frac{\partial u_{i}}{\partial t}=-\beta_{i} \frac{\delta}{\delta u_{i}} \mathcal{H}(u, \lambda)+\Phi_{i}(u, \nabla u, \lambda) \quad \text { for } 1 \leq i \leq m \\
& \left.\frac{\partial u}{\partial n}\right|_{\partial \Omega}=0 \quad\left(\text { or }\left.u\right|_{\partial \Omega}=0\right),
\end{aligned}
$$

where $\delta / \delta u_{i}$ are the variational derivative, $\beta_{i}>0$ and $\Phi_{i}$ satisfy

$$
\int_{\Omega} \sum_{i} \Phi_{i} \frac{\delta}{\delta u_{i}} \mathcal{H}(u, \lambda) d x=0 .
$$

The condition 2.9) is required by the Le Châtelier principle. In the concrete problem, the terms $\Phi_{i}$ can be determined by physical laws and 2.9 . We remark here that following the le Châtelier principle, one should have an inequality constraint. However physical systems often obey most simplified rules, as many existing models for specific problems are consistent with the equality constraint here. This remark applies to the constraint 2.15) below as well.

When the order parameters are the number density and the system has no material exchange with the external, then $u_{j}(1 \leq j \leq m)$ are conserved, i.e.,

$$
\int_{\Omega} u_{j}(x, t) d x=\text { constant. }
$$

This conservation law requires a continuity equation

$$
\frac{\partial u_{j}}{\partial t}=-\nabla \cdot J_{j}(u, \lambda)
$$


where $J_{j}(u, \lambda)$ is the flux of component $u_{j}$. In addition, $J_{j}$ satisfy

$$
J_{j}=-k_{j} \nabla\left(\mu_{j}-\sum_{i \neq j} \mu_{i}\right),
$$

where $\mu_{l}$ is the chemical potential of component $u_{l}$,

$$
\mu_{j}-\sum_{i \neq j} \mu_{i}=\frac{\delta}{\delta u_{j}} \mathcal{H}(u, \lambda)-\phi_{j}(u, \nabla u, \lambda),
$$

and $\phi_{j}(u, \lambda)$ is a function depending on the other components $u_{i}(i \neq j)$. Thus, from (2.11)-2.13) we obtain the dynamical equations as follows

$$
\begin{aligned}
& \frac{\partial u_{j}}{\partial t}=\beta_{j} \Delta\left[\frac{\delta}{\delta u_{j}} \mathcal{H}(u, \lambda)-\phi_{j}(u, \nabla u, \lambda)\right] \quad \text { for } 1 \leq j \leq m, \\
& \left.\frac{\partial u}{\partial n}\right|_{\partial \Omega}=0,\left.\quad \frac{\partial \Delta u}{\partial n}\right|_{\partial \Omega}=0
\end{aligned}
$$

where $\beta_{j}>0$ are constants, and $\phi_{j}$ satisfy

$$
\int_{\Omega} \sum_{j} \Delta \phi_{j} \cdot \frac{\delta}{\delta u_{j}} \mathcal{H}(u, \lambda) d x=0 .
$$

When $m=1$, i.e., the system is a binary system, consisting of two components $A$ and $B$, then the term $\phi_{j}=0$. The above model covers the classical Cahn-Hilliard model. It is worth mentioning that for multi-component systems, these $p h i_{j}$ play an important rule in deriving good time-dependent models.

If the order parameters $\left(u_{1}, \cdots, u_{k}\right)$ are coupled to the conserved variables $\left(u_{k+1}, \cdots, u_{m}\right)$, then the dynamical equations are

$$
\begin{array}{ll}
\frac{\partial u_{i}}{\partial t}=-\beta_{i} \frac{\delta}{\delta u_{i}} \mathcal{H}(u, \lambda)+\Phi_{i}(u, \nabla u, \lambda) & \text { for } 1 \leq i \leq k, \\
\frac{\partial u_{j}}{\partial t}=\beta_{j} \Delta\left[\frac{\delta}{\delta u_{j}} \mathcal{H}(u, \lambda)-\phi_{j}(u, \nabla u, \lambda)\right] & \text { for } k+1 \leq j \leq m, \\
\left.\frac{\partial u_{i}}{\partial n}\right|_{\partial \Omega}=0 \quad\left(\text { or }\left.u_{i}\right|_{\partial \Omega}=0\right) & \text { for } 1 \leq i \leq k, \\
\left.\frac{\partial u_{j}}{\partial n}\right|_{\partial \Omega}=0,\left.\quad \frac{\partial \Delta u_{j}}{\partial n}\right|_{\partial \Omega}=0 & \text { for } k+1 \leq j \leq m .
\end{array}
$$

Here $\Phi_{i}$ and $\phi_{j}$ satisfy (2.9) and 2.15), respectively.

The model (2.16) we derive here gives a general form of the governing equations to thermodynamic phase transitions, and will play crucial role in studying the dynamics of equilibrium phase transition in statistic physics.

\section{Dynamic Model for Liquid ${ }^{4} \mathrm{He}$}

${ }^{4} \mathrm{He}$ was first liquidized at $T=4.215 K$ and $p=1 \times 10^{5}(\mathrm{~Pa})$ by Karmerlingh Onnes in 1908. In 1938, P.L.Kapitza found that when the temperature $T$ decreases below $T_{C}=2.17 \mathrm{~K}$, the liquid ${ }^{4} \mathrm{He}$ will transit from normal liquid state to superfluid state, in which the fluid possesses zero viscosity i.e., the viscous coefficient $\eta=0$. The liquids with $\eta=0$ are called the superfluids, and the flow without drag is called the superfluidity. The superfluid transition is called $\lambda$-phase transition, and its phase diagram is illustrated by Figure 3.1 . 


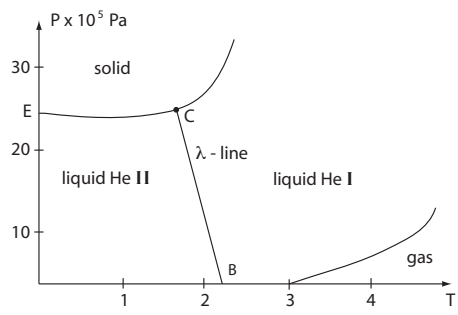

Figure 3.1. Classical $P T$ phase diagram of ${ }^{4} \mathrm{He}$.

3.1. Ginzburg-Landau free energy. The order parameter describing superfluidity is characterized by a non vanishing complex valued function $\psi: \Omega \rightarrow \mathbb{C}$ as in the superconductivity, originating from quantum Bose-Einstein condensation. In the two-fluid hydrodynamic theory of super-fluidity, the density $\rho$ of ${ }^{4} \mathrm{He}$ is given by

$$
\rho=\rho_{s}+\rho_{n},
$$

where $\rho_{s}$ is the superfluid density and $\rho_{n}$ the normal fluid density. The square $|\psi|^{2}$ is proportional to $\rho_{s}$, and without loss of generality, we take $\psi$ as

$$
|\psi|^{2}=\rho_{s}
$$

Based on the quantum mechanics, $-i h \nabla \psi$ represents the momentum associated with the Bose-Einstein condensation. Hence, the free energy density contains the term

$$
\frac{1}{2 m}|-i h \nabla \psi|^{2}=\frac{h^{2}}{2 m}|\nabla \psi|^{2}
$$

where $h$ is the Planck constant, and $m$ the mass of atom ${ }^{4} \mathrm{He}$. Meanwhile the superfluid state does not obey the classical thermodynamic laws, which the normal liquid state obeys. Therefore, in the free energy density, $\psi$ satisfies the GinzburgLandau expansion

$$
\frac{k_{1} h^{2}}{2 m}|\nabla \psi|^{2}+\frac{\gamma_{1}}{2}|\psi|^{2}+\frac{\gamma_{2}}{4}|\psi|^{4},
$$

and $\rho_{n}$ has the expansion as in the free energy for PVT systems [4. For simplicity we ignore the entropy, and consider the coupling action of $\psi$ and $\rho_{n}$, i.e., add the term $\frac{1}{2} \mu \rho_{n}|\psi|^{2}$ in the free energy density. Thus the Ginzburg-Landau free energy for liquid ${ }^{4} \mathrm{He}$ near the superfluid transition is given by

$$
\begin{aligned}
G\left(\psi, \rho_{n}\right)= & \int_{\Omega}\left[\frac{k_{1} h^{2}}{2 m}|\nabla \psi|^{2}+\frac{\gamma_{1}}{2}|\psi|^{2}+\frac{\gamma_{2}}{4}|\psi|^{4}+\frac{\gamma_{3}}{2} \rho_{n}|\psi|^{2}\right. \\
& \left.+\frac{k_{2}}{2}\left|\nabla \rho_{n}\right|^{2}+\frac{\mu_{1}}{2} \rho_{n}^{2}+\frac{\mu_{2}}{3} \rho_{n}^{3}+\frac{\mu_{3}}{4} \rho_{n}^{4}-p\left(\rho_{n}+\frac{\mu_{0}}{2} \rho_{n}^{2}\right)\right] d x .
\end{aligned}
$$

3.2. Dynamic model governing the superfluidity. By (2.8), we derive from (3.3) the following time-dependent Ginzburg-Landau equations governing the superfluidity of liquid ${ }^{4} \mathrm{He}$ :

$$
\begin{aligned}
& \frac{\partial \psi}{\partial t}=\frac{k_{1} h^{2}}{m} \Delta \psi-\gamma_{1} \psi-\gamma_{2}|\psi|^{2} \psi-\gamma_{3} \rho_{n} \psi, \\
& \frac{\partial \rho_{n}}{\partial t}=k_{2} \Delta \rho_{n}-\left(\mu_{1}-p \mu_{0}\right) \rho_{n}-\mu_{2} \rho_{n}^{2}-\mu_{3} \rho_{n}^{3}-\frac{\gamma_{3}}{2}|\psi|^{2}+p .
\end{aligned}
$$


It is known that the following problem has a solution $\rho_{n}^{0} \in H^{2}(\Omega) \cap H_{0}^{1}(\Omega)$ standing for the density of liquid He I for any $p \in L^{2}(\Omega)$ :

$$
\begin{aligned}
& -k_{2} \Delta \rho_{n}^{0}+\left(\mu_{1}-p \mu_{0}\right) \rho_{n}^{0}+\mu_{2}\left(\rho_{n}^{0}\right)^{2}+\mu_{3}\left(\rho_{n}^{0}\right)^{3}=p \\
& \left.\frac{\partial \rho_{n}^{0}}{\partial n}\right|_{\partial \Omega}=0 .
\end{aligned}
$$

To derive the nondimensional form of (3.4), let

$$
\begin{array}{ll}
(x, t)=\left(l x^{\prime}, \tau t^{\prime}\right), & \left(\psi, \rho_{n}\right)=\left(\psi_{0} \psi^{\prime}, \rho_{0} \rho_{n}^{\prime}+\rho_{n}^{0}\right), \\
\tau=\frac{m l^{2}}{h^{2} k_{1}}, & \mu=\frac{k_{2} \tau}{l^{2}}, \\
a_{1}=\gamma_{3} \rho_{0} \tau, & a_{2}=\gamma_{2}\left|\psi_{0}\right|^{2} \tau, \\
b_{1}=\frac{\gamma_{3}\left|\psi_{0}\right|^{2}}{2 \rho_{0}} \tau, & b_{2}=\tau \rho_{0}\left(3 \rho_{n}^{0} \mu_{3}+\mu_{2}\right), \\
b_{3}=\rho_{0}^{2} \mu_{3} \tau, & \lambda_{1}=-\tau\left(\gamma_{1}+\gamma_{3} \rho_{n}^{0}\right), \\
\lambda_{2}=\tau\left(3\left(\rho_{n}^{0}\right)^{2} \mu_{3}+2 \rho_{n}^{0} \mu_{2}+\mu_{0} p-\mu_{1}\right), &
\end{array}
$$

where $\rho_{n}^{0}$ is the solution of 3.5 .

Thus, suppressing the primes, the equations (3.4) are rewritten as

$$
\begin{aligned}
& \frac{\partial \psi}{\partial t}=\Delta \psi+\lambda_{1} \psi-a_{1} \rho_{n} \psi-a_{2}|\psi|^{2} \psi, \\
& \frac{\partial \rho_{n}}{\partial t}=\mu \Delta \rho_{n}+\lambda_{2} \rho_{n}-b_{1}|\psi|^{2}-b_{2} \rho_{n}^{2}-b_{3} \rho_{n}^{3} .
\end{aligned}
$$

The boundary conditions associated with (3.6) are

$$
\frac{\partial \psi}{\partial n}=0, \quad \frac{\partial \rho_{n}}{\partial n}=0 \quad \text { on } \partial \Omega .
$$

When the pressure $p$ is independent of $x \in \Omega$, then the problem (3.6) and (3.7) can be approximatively replaced by the following systems of ordinary differential equations for superfluid transitions:

$$
\begin{aligned}
& \frac{d \psi}{d t}=\lambda_{1} \psi-a_{1} \rho_{n} \psi-a_{2}|\psi|^{2} \psi, \\
& \frac{d \rho_{n}}{d t}=\lambda_{2} \rho_{n}-b_{1}|\psi|^{2}-b_{2} \rho_{n}^{2}-b_{3} \rho_{n}^{3} .
\end{aligned}
$$

By multiplying $\psi^{*}$ to both sides of the first equation of $(3.8)$ and by $(3.2)$, the equations (3.8) are reduced to:

$$
\begin{aligned}
& \frac{d \rho_{s}}{d t}=\lambda_{1} \rho_{s}-a_{1} \rho_{n} \rho_{s}-a_{2} \rho_{s}^{2}, \\
& \frac{d \rho_{n}}{d t}=\lambda_{2} \rho_{n}-b_{1} \rho_{s}-b_{2} \rho_{n}^{2}-b_{3} \rho_{n}^{3}, \\
& \left(\rho_{s}(0), \rho_{n}(0)\right)=\left(x_{0}, y_{0}\right) .
\end{aligned}
$$

for $\rho_{s} \geq 0$ and $x_{0} \geq 0$.

We need to explain the physical properties of the coefficients in (3.4) and (3.6). It is known that the coefficients $\gamma_{i}(1 \leq i \leq 3)$ and $\mu_{j}(0 \leq j \leq 3)$ depend continuously on the temperature $T$ and the pressure $p$ :

$$
\gamma_{i}=\gamma_{i}(T, p), \quad \mu_{j}=\mu_{j}(T, p) \quad \forall 1 \leq i \leq 3, \quad 0 \leq j \leq 3 .
$$


From the both mathematical and physical points of view, the following conditions are required:

$$
\gamma_{2}>0, \quad \gamma_{3}>0, \quad \mu_{3}>0 \quad \forall T, \rho .
$$

In addition, by the Landau mean field theory we have

$$
\begin{gathered}
\gamma_{1} \begin{cases}>0 & \text { if }(T, p) \in A_{1}, \\
<0 & \text { if }(T, p) \in A_{2},\end{cases} \\
\mu_{1} \begin{cases}>0 & \text { if }(T, p) \in B_{1}, \\
<0 & \text { if }(T, p) \in B_{2},\end{cases}
\end{gathered}
$$

where $A_{i}, B_{i}(i=1,2)$ are connected open sets such that $\bar{A}_{1}+\bar{A}_{2}=\bar{B}_{1}+\bar{B}_{2}=\mathbb{R}_{+}^{2}$, and $\bar{A}_{1} \cap \bar{A}_{2}, \bar{B}_{1} \cap \bar{B}_{2}$ are two simple curves in $\mathbb{R}_{+}^{2}$; see Figure 4.1 (a) and (b). In particular, in the PT-plane,

$$
\frac{\partial \gamma_{1}}{\partial T}>0, \quad \frac{\partial \gamma_{1}}{\partial p}>0, \quad \frac{\partial \mu_{1}}{\partial T}>0, \quad \frac{\partial \mu_{1}}{\partial p}>0 .
$$

By 3.10 the following nondimensional parameters are positive

$$
a_{1}>0, \quad a_{2}>0, \quad b_{1}>0, \quad b_{3}>0 .
$$

By (3.11)-(3.13), the following two critical parameter equations

$$
\begin{aligned}
& \lambda_{1}=\lambda_{1}(T, p)=0, \\
& \lambda_{2}=\lambda_{2}(T, p)=0,
\end{aligned}
$$

give two simple curves $l_{1}$ and $l_{2}$ respectively in the $P T$-plane $\mathbb{R}_{+}^{2}$; see Figure 4.1 (c) and (d).

The parameters $\mu_{2}$ and $b_{2}$ depend on the physical properties of the atom He, and satisfy the following relations:

$$
\begin{aligned}
& b_{2}(T, p)=\tau \rho_{0}\left(\mu_{2}+3 \rho_{n}^{0} \mu_{3}\right)<0 \quad \text { iff } \rho_{\text {sol }}>\rho_{l} \text { at } \lambda_{2}(T, p)=0, \\
& b_{2}(T, p)=\tau \rho_{0}\left(\mu_{2}+3 \rho_{n}^{0} \mu_{3}\right)>0 \quad \text { iff } \rho_{\text {sol }}<\rho_{l} \text { at } \lambda_{2}(T, p)=0,
\end{aligned}
$$

where $\rho_{\text {sol }}$ and $\rho_{l}$ are the densities of solid and liquid, and $\rho_{n}^{0}$ the solution of 3.5 representing the liquid density. These relations in $(3.16)$ can be deduced by the dynamic transition theorem of (2.1) from a simple critical eigenvalue, Theorem A.2 in Ma and Wang $[5$.

\section{Dynamic Phase transition for Liquid ${ }^{4} \mathrm{He}$}

In order to illustrate the main ideas, we discuss only the case where the pressure $p$ is independent of $x \in \Omega$, i.e., we only consider the equations (3.8) and (3.9); the general case can be studied in the same fashion and will be reported elsewhere.

4.1. PT-phase diagram. Based on physical experiments together with (3.11)(3.13), the curves of $\gamma_{1}(T, p)=0$ and $\mu_{1}(T, p)=0$ in the $P T$-plane are given by Figure 4.1(a) and (b) respectively. By the formulas

$$
\begin{aligned}
& \lambda_{1}(T, p)=-\tau\left(\gamma_{1}(T, p)+\gamma_{3} \rho_{n}^{0}\right), \\
& \lambda_{2}(T, p)=-\tau\left(\mu_{1}(T, p)-\mu_{0} p-2 \rho_{n}^{0} \mu_{2}-3 \rho_{n}^{02} \mu_{3}\right),
\end{aligned}
$$

together with (3.10) and (3.16), the curves $l_{1}$ and $l_{2}$ given by (3.15) in the $P T$-plane are illustrated in Figure 4.1(c) and (d). 

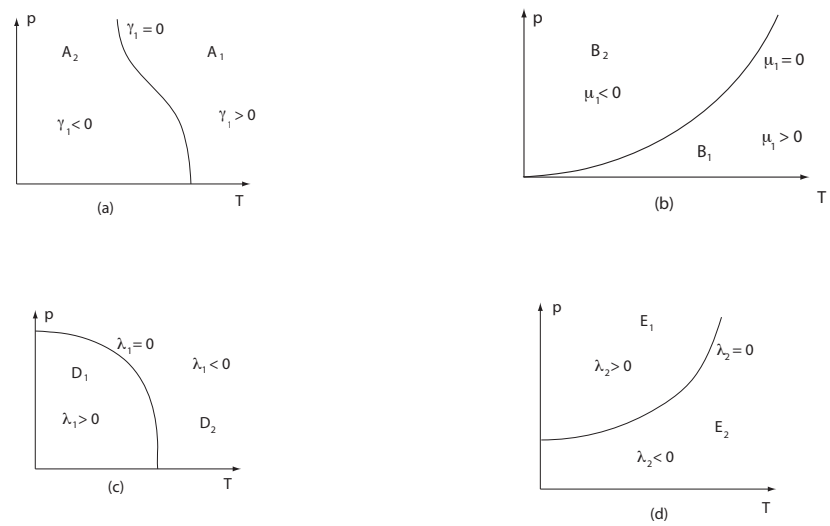

FiguRE 4.1. (a) The curve of $\gamma_{1}=0$, (b) the curve of $\mu_{1}=0$, (c) the curve of $\lambda_{1}=0,(\mathrm{~d})$ the curve of $\lambda_{2}=0$.

Figure 4.2. $A C F$ is the curve of $\lambda_{2}=0, B C D$ is the curve of $\lambda_{1}=$ 0.

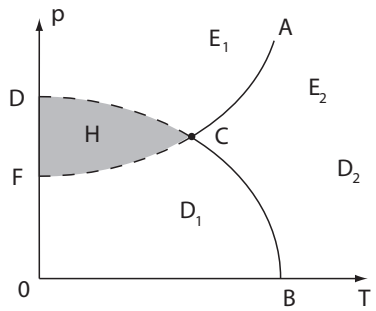

Let

$$
\begin{aligned}
& D_{1}=\left\{(T, p) \in \mathbb{R}_{+}^{2} \mid \lambda_{1}(T, p)>0\right\}, \\
& D_{2}=\left\{(T, p) \in \mathbb{R}_{+}^{2} \mid \lambda_{1}(T, p)<0\right\}, \\
& E_{1}=\left\{(T, p) \in \mathbb{R}_{+}^{2} \mid \lambda_{2}(T, p)>0\right\}, \\
& E_{2}=\left\{(T, p) \in \mathbb{R}_{+}^{2} \mid \lambda_{2}(T, p)<0\right\} .
\end{aligned}
$$

Let the curve of $\lambda_{1}(T, p)=0$ intersect with the curve of $\lambda_{2}(T, p)=0$ at a point $C$; see Figure 4.2 . 
When $\lambda_{1}$ crosses the curve segment $C B$ to enter into $D_{1}$ from $D_{2}$ (see Figure 4.2 , we have $\lambda_{2}<0$ and $\lambda_{1}$ satisfies

$$
\lambda_{1}(T, p) \begin{cases}<0 & \text { if } \quad(T, p) \in D_{2}, \\ =0 & \text { if } \quad(T, p) \in C B, \\ >0 & \text { if } \quad(T, p) \in D_{1} .\end{cases}
$$

In this case, by Theorem 2.1. the equations $(3.9)$ have a phase change which describes the transition from liquid He-I to liquid He-II, and the second equation of (3.9) can be equivalently rewritten as

$$
\frac{d \widetilde{\rho_{n}}}{d t}=\left(\lambda_{2}-2 b_{2} \rho_{n}^{*}-3 b_{3}\left(\rho_{n}^{*}\right)^{2}\right) \widetilde{\rho_{n}}-\left(b_{2}+3 b_{3} \rho_{n}^{*}\right) \widetilde{\rho}_{n}^{2}-b_{3} \widetilde{\rho}_{n}^{3}
$$

where $\widetilde{\rho}_{n}=\rho_{n}-\rho_{n}^{*}, \rho_{n}^{*}$ satisfies the equation

$$
\lambda_{2} \rho_{n}-b_{2} \rho_{n}^{2}-b_{3} \rho_{n}^{3}=b_{1} \rho_{s},
$$

and $\rho_{s}>0$ is the transition solution of $(3.9)$. Since $\rho_{n}^{*}$ is the density deviation of normal liquid, by (3.1) we have

$$
\rho_{n}^{*}=\rho-\rho_{n}^{0}-\rho_{s} .
$$

On the other hand, $\rho_{n}^{0}(\simeq \rho)$ represents the density of liquid He I. Thus, $\rho_{n}^{*} \simeq$ $-\rho_{s}<0$. From the $P T$-phase diagram of ${ }^{4} \mathrm{He}$ (Figure 3.1), we see that $\rho_{\text {sol }} \geq \rho_{l}$ for ${ }^{4} \mathrm{He}$ near $T=0$, therefore by $(3.16), b_{2} \leq 0$. Hence we derive that

$$
\lambda_{2}-2 b_{2} \rho_{n}^{*}-3 b_{3}\left(\rho_{n}^{*}\right)^{2}<0,
$$

for $(T, p)$ in the region $D_{1} \backslash H$, as shown in Figure 4.2 .

It follows from (4.1) and (4.2) that when $(T, p)$ is in the region $D_{1} \backslash H$, the liquid ${ }^{4} \mathrm{He}$ is in the superfluid state.

When $\lambda_{2}$ crosses the curve segment $C A$, as shown in Figure 4.2 , to enter into $E_{1}$ from $E_{2}$, then $\lambda_{1}<0$ and

$$
\lambda_{2}(T, p) \begin{cases}<0 & \text { if } \quad(T, p) \in E_{2}, \\ =0 & \text { if } \quad(T, p) \in C A, \\ >0 & \text { if } \quad(T, p) \in E_{1} .\end{cases}
$$

In this case, the equations $(3.9)$ characterize the liquid-solid phase transition, and the first equation of $(3.9)$ is equivalently expressed as

$$
\frac{d \rho_{s}}{d t}=\left(\lambda_{1}-a_{1} \widetilde{\rho}_{n}\right) \rho_{s}-a_{2} \rho_{s}^{2},
$$

where $\widetilde{\rho}_{n}>0$ is the transition state of solid ${ }^{4} \mathrm{He}$. By 3.14 we have

$$
\lambda_{1}(T, p)-a_{1} \widetilde{\rho}_{n}<0,
$$

for any $(T, p)$ in the region $E_{1} \backslash H$, as shown in Figure 4.2

From (4.3) and (4.4) we can derive the conclusion that as $(T, p)$ in $E_{1} \backslash H, \rho_{s}=0$ is stable, i.e., ${ }^{4} \mathrm{He}$ is in the solid state.

However, the shadowed region $H$ in Figure 4.2 is an unstable domain for the solid and liquid He II states, where any of these two phases may appear depending on the random fluctuations. Thus, from the discussion above, we can derive the theoretical $P T$-phase diagram given by Figure 4.3 , based on equations 3.9 . In comparison with the experimental $P T$-phase diagram (Figure 3.1), a sight difference in Figure 4.3 is that there exists an unstable region $H$, where the solid phase and the He II 
Figure 4.3. Theoretical PTphase diagram: $H$ is an unstable region where both solid and liquid He II states appear randomly depending on fluctuations. The point $M$ is a point where the transitions, between superfluid state (liquid He II) and the normal fluid state (liquid He I), changes from Type II to Type-I; namely, the transition crossing $C M$ is TypeII and the transition crossing $M B$ is Type-I.

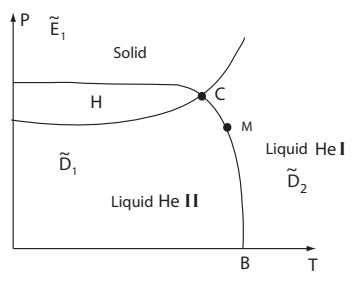

phase are possible to occur. This unstable region in Figure 4.3 corresponding to the coexistence curve $C E$ in Figure 3.1 is a theoretical prediction, which need to be verified by experiments.

4.2. States in unstable region. We consider the dynamical properties of transitions for $(3.9)$ in the unstable region. It is clear that at point $C=\left(T_{C}, p_{C}\right)$,

$$
\lambda_{1}\left(T_{C}, p_{C}\right)=0, \quad \lambda_{2}\left(T_{C}, p_{C}\right)=0,
$$

and the unstable region $H$ satisfies that

$$
H=\left\{(T, p) \in \mathbb{R}_{+}^{2} \mid \lambda_{1}(T, p)>0, \lambda_{2}(T, p)>0\right\} .
$$

To study the structure of flows of $(3.9)$ for $(T, p) \in H$ it is necessary to consider the equations $(3.9)$ at the point $C=\left(T_{C}, p_{C}\right)$, and by 4.5 which are given by

$$
\begin{aligned}
& \frac{d \rho_{s}}{d t}=-a_{1} \rho_{n} \rho_{s}-a_{2} \rho_{s}^{2}, \\
& \frac{d \rho_{n}}{d t}=-b_{1} \rho_{s}-b_{2} \rho_{n}^{2}-b_{3} \rho_{n}^{3} .
\end{aligned}
$$

Due to (3.16) and $\rho_{\text {sol }}>\rho_{l}$, we have

$$
b_{2}<0 \quad \text { for } \quad(T, p) \subset H .
$$

Under the condition (4.7), equations 4.6 have the following two steady state solutions:

$$
\begin{aligned}
& Z_{1}=\left(\rho_{s}, \rho_{n}\right)=\left(0,\left|b_{2}\right| / b_{3}\right), \\
& Z_{2}=\left(\rho_{s}, \rho_{n}\right)=\left(\frac{a_{1}}{a_{2}} \alpha,-\alpha\right), \\
& \alpha=\frac{\left|b_{2}\right|}{2 b_{3}}\left(\sqrt{1+4 a_{1} b_{1} b_{3} / a_{2}\left|b_{2}\right|^{2}}-1\right) .
\end{aligned}
$$

By direct computation, we can prove that the eigenvalues of the Jacobian matrices of (4.6) at $Z_{1}$ and $Z_{2}$ are negative. Hence, $Z_{1}$ and $Z_{2}$ are stable equilibrium points of (4.6). Physically, $Z_{1}$ stands for solid state, and $Z_{2}$ for superfluid state. The topological structure of 4.6 is schematically illustrated by Figure 4.4 (a), the two regions $R_{1}$ and $R_{2}$ divided by curve $A O$ in Figure $4.4(\mathrm{~b})$ are the basins of attraction of $Z_{1}$ and $Z_{2}$ respectively. 
We note that in $H, \lambda_{1}$ and $\lambda_{2}$ is small, i.e.,

$$
0<\lambda_{1}(T, p), \quad \lambda_{2}(T, p) \ll 1, \quad \text { for }(T, p) \in H,
$$

and (3.9) can be consider as a perturbed system of 4.6.

Thus, for $(T, p) \in H$ the system 3.9 have four steady state solutions $\widetilde{Z}_{i}=$ $\widetilde{Z}(T, p)(1 \leq i \leq 4)$ such that

$$
\lim _{(T, p) \rightarrow\left(T_{C}, p_{C}\right)}\left(\widetilde{Z}_{1}(T, p), \widetilde{Z}_{2}(T, p), \widetilde{Z}_{3}(T, p), \widetilde{Z}_{4}(T, p)\right)=\left(Z_{1}, Z_{2}, 0,0\right),
$$

and $\widetilde{Z}_{1}$ and $\widetilde{Z}_{2}$ are stable, representing solid state and liquid He-II state respectively, $\widetilde{Z}_{3}$ and $\widetilde{Z}_{4}$ are two saddle points. The topological structure of 3.9 for $(T, p) \in H$ is schematically shown in Figure $4.4(\mathrm{c})$, and the basins of attraction of $\widetilde{Z}_{1}$ and $\widetilde{Z}_{2}$ are $\widetilde{R}_{1}$ and $\widetilde{R}_{2}$ as illustrated by Figure 4.4 (d).

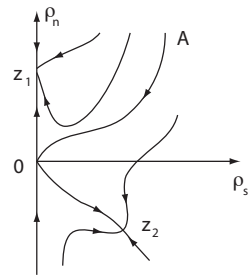

(a)

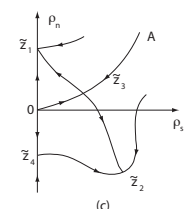

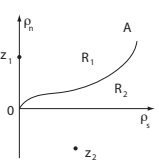

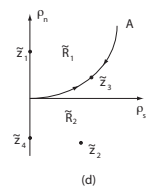

FIGURE 4.4 .

In summary, with the above analysis and the dynamic transition theory, we arrive at the following theorem:

Theorem 4.1. There exist four regions $\widetilde{E}_{1}, \widetilde{D}_{1}$ and $H$ in the PT-plane (see Figure 4.3), which are defined by

$$
\begin{aligned}
& \widetilde{E}_{1}=\left\{(T, p) \in \mathbb{R}_{+}^{2} \mid \lambda_{1}(T, p)<0, \lambda_{2}(T, p)>0\right\}, \\
& \widetilde{D}_{1}=\left\{(T, p) \in \mathbb{R}_{+}^{2} \mid \lambda_{1}(T, p)>0, \lambda_{2}(T, p)<0\right\}, \\
& \tilde{D}_{2}=\left\{(T, p) \in \mathbb{R}_{+}^{2} \mid \lambda_{1}(T, p)<0, \lambda_{2}(T, p)<0\right\}, \\
& H=\left\{(T, p) \in \mathbb{R}_{+}^{2} \mid \lambda_{1}(T, p)>0, \lambda_{2}(T, p)>0\right\},
\end{aligned}
$$

such that the following conclusions hold true:

(1) If $(T, p) \in \widetilde{E}_{1}$, the phase of ${ }^{4}$ He is in solid state.

(2) If $(T, p) \in \widetilde{D}_{1}$, the phase is in superfluid state.

(3) If $(T, p) \in \widetilde{D}_{2}$, the phase is in the normal fluid state. 
(4) If $(T, p) \in H$, there are two regions $\widetilde{R}_{1}$ and $\widetilde{R}_{2}$ in the state space $\left(\rho_{s}, \rho_{n}\right)$ such that, under a fluctuation which is described by the initial value $\left(x_{0}, y_{0}\right)$ in (3.9), if $\left(x_{0}, y_{0}\right) \in \widetilde{R}_{1}$ then the phase is in solid state, and if $\left(x_{0}, y_{0}\right) \in \widetilde{R}_{2}$ then it is in superfluid state.

In general, the observed superfluid transitions of ${ }^{4} \mathrm{He}$ are of the second order, i.e., Type-I (continuous) with the dynamic classification. But, from $(3.9)$ we can prove the following theorem, which shows that for a higher pressure the superfluid transitions may be of zeroth order, i.e., the Type-II (jump) transition with the dynamic classification scheme.

Theorem 4.2. Let $\left(T_{0}, p_{0}\right)$ satisfy that $\lambda_{1}\left(T_{0}, \rho_{0}\right)=0, \lambda_{2}\left(T_{0}, p_{0}\right)<0$. Then, (3.9) have a superfluid transition at $\left(T_{0}, p_{0}\right)$ from $D_{2}$ to $D_{1}$. In particular, the following assertions hold true:

(1) Let

$$
A=\frac{a_{1} b_{1}}{\left|\lambda_{2}\right|}-a_{2} \quad \text { at }(T, p)=\left(T_{0}, p_{0}\right) .
$$

Then if $A<0$ the superfluid transition is Type-I, and if $A>0$ the superfluid transition is Type-II.

(2) The equation

$$
A=\frac{a_{1} b_{1}}{\left|\lambda_{2}\right|}-a_{2}=0
$$

determines a point $M$ on the $C B$ coexistence curve in Figure 4.3, where the transition changes type from Type-II to Type-I.

Proof. STEP 1. By the assumption, it is clear that

$$
\lambda_{1}(T, p)= \begin{cases}<0 & \text { if } \quad(T, p) \in D_{2}, \\ =0 & \text { if }(T, p)=\left(T_{0}, p_{0}\right), \\ >0 & \text { if } \quad(T, p) \in D_{1},\end{cases}
$$

where $D_{1}, D_{2}$ are as in Figure 4.1 (c). Hence, by Theorem 2.1, (3.9) have a transition at $\left(T_{0}, p_{0}\right)$. By $\lambda_{2}\left(T_{0}, p_{0}\right)<0$, the first eigenvalue of $(3.9)$ is simple, and its eigenvector is given by $e=\left(e_{s}, e_{n}\right)=(1,0)$.

The reduced equation of (3.9) on the center manifold reads

$$
\frac{d x}{d t}=\lambda_{1} x-a_{1} x h(x)-a_{2} x^{2}, \quad x>0,
$$

where $h(x)$ is the center manifold function. By $[3.9), h$ can be expressed as

$$
h(x)=\frac{b_{1}}{\lambda_{2}} x+o\left(x^{2}\right), \quad x>0 .
$$

Thus the reduced equation of 3.9 is given by

$$
\frac{d x}{d t}=\lambda_{1} x+A x^{2}+o\left(x^{2}\right) \quad \text { for } x>0,
$$

where $A$ is as in 4.8. The theorem follows from 4.9) and Theorem 2.2

SteP 2. By the nondimensional form, we see that

$$
a_{1} b_{1}=\frac{1}{2} \gamma_{3}^{2}\left|\psi_{0}\right|^{2} \tau^{2}
$$


where $\gamma_{3}$ is the coupled coefficient of $\rho_{n}$ and $\rho_{s}$. Physically, $\gamma_{3}$ is small in comparison with $\gamma_{2}$; namely

$$
0<a_{1} b_{1} \ll a_{2} .
$$

On the other hand, we know that

$$
\lambda_{2}\left(T_{0}, p_{0}\right) \rightarrow 0 \quad \text { as } \quad\left(T_{0}, p_{0}\right) \rightarrow\left(T_{C}, p_{C}\right),
$$

where $C=\left(T_{C}, p_{C}\right)$ is as in 4.5 . Therefore we deduce that there exists a pressure $p^{*}\left(b<p^{*}<p_{C}\right)$ such that

$$
A=\frac{a_{1} b_{1}}{\left|\lambda_{2}\left(T_{0}, p_{0}\right)\right|}-a_{2} \begin{cases}<0 & \text { if } 0 \leq p_{0}<p^{*} \\ >0 & \text { if } p^{*}<p_{0}<p_{C}\end{cases}
$$

Thus, when the transition pressure $p_{0}$ is below some value $p_{0}<p^{*}$ the superfluid transition is of the second order, i.e., is continuous with the dynamic classification scheme, and when $p_{0}<p^{*}$ the superfluid transition is the first order, i.e., is jump in the dynamic classification scheme. The proof is complete.

\section{Physical Conclusions and Predictions}

As we know, the classical phase transition is illustrated by Figure 3.1. In this phase diagram, the coexistence curve $C E$ separates the solid state and the superfluid state (liquid He II), and the curve $C B$ is the coexistence curve between the superfluid state (liquid He II) and the normal fluid state (liquid He I), and the critical point is the triple-point. It is considered by the classical theory that the transition crossing $C B$ is second order in the Ehrenfest sense (Type-I with the dynamic classification scheme).

However, in the phase transition diagram Figure 4.3 derived based on Theorems 4.1 and 4.2 , there is a unstable region $H$, where both solid and liquid He II states appear randomly depending on fluctuations. The point $M$ is a switch point where the transitions, between superfluid state (liquid He II) and the normal fluid state (liquid He I), changes from first order (Type II with the dynamic classification scheme) to second order (Type-I); namely, the transition crossing $C M$ is second order (Type-II) and the transition crossing $M B$ is first order (Type-I).

In summary, the results in this article predict the existence of the unstable region $H$ and the existence of the switch point $M$. It is hoped these predictions can be verified by experiments.

\section{REFERENCES}

[1] V. L. GinzBurg, On superconductivity and superfluidity (what $i$ have and have not managed to do), as well as on the 'physical minimum' at the beginning of the xxi century, Phys.-Usp., 47 (2004), pp. 1155-1170.

[2] T. MA AND S. WANG, Bifurcation theory and applications, vol. 53 of World Scientific Series on Nonlinear Science. Series A: Monographs and Treatises, World Scientific Publishing Co. Pte. Ltd., Hackensack, NJ, 2005.

[3] - Stability and Bifurcation of Nonlinear Evolutions Equations, Science Press, 2007.

[4] — Dynamic phase transition theory in PVT systems, Indiana University Mathematics Journal, to appear; see also Arxiv: 0712.3713, (2008).

[5] — Dynamic phase transitions for ferromagnetic systems, Journal of Mathematical Physics, 49:053506 (2008), pp. 1-18.

[6] O. Onuki, Phase transition dynamics, Combridge Univ. Press., (2002).

[7] L. E. ReIchl, A modern course in statistical physics, A Wiley-Interscience Publication, John Wiley \& Sons Inc., New York, second ed., 1998. 
(TM) Department of Mathematics, Sichuan University, Chengdu, P. R. China

(SW) Department of Mathematics, Indiana University, Bloomington, IN 47405

E-mail address: showang@indiana.edu, http://www.indiana.edu/ fluid 